\title{
Prediction of liquid densities for halocarbon mixtures by a modified Hard Sphere-De Santis equation of state
}

\author{
Paulo S. Fialho ${ }^{\text {a, } *}$, C.A. Nieto de Castro ${ }^{b}$ \\ "Departamento de Ciências Agrárias, Universidade dos Açores, 9702 Angra do Heroísmo Codex, Portugal \\ ${ }^{\mathrm{h}}$ Departamento de Química and ICAT, Faculdade de Ciências da Universidade de Lisboa, Edifício Cl, Piso 5, Campo \\ Grande, 1700 Lisboa, Portugal
}

Received 7 May 1995; accepted 30 July 1995

\begin{abstract}
In a companion paper, we dealt with the problem of predicting the liquid behaviour of pure refrigerants. It is the purpose of the present paper to extend the use of the Hard Sphere De Santis (HSDS) model by associating it with mixing and combination rules to predict the liquid state densities for mixtures of halocarbon refrigerants.

The deviations obtained between the experimental data, available for binary and ternary mixtures, and the HSDS model have shown an uncertainty of $1.5 \%$ for $T^{*} \leq 0.9$. This was the uncertainty claimed for the model, when applied to pure fluids.
\end{abstract}

Keywords: Theory; Corresponding states; Equation of state; Density; Halocarbons; Liquid mixtures

\section{Introduction}

As stated before (Fialho and Nieto de Castro, 1995) the increasing need for replacement refrigerants less harmful to the environment is done not only by replacing the CFC pure fluids by the HCFCs and HFCs, but also by mixtures of them. The aim is to obtain the best properties of the CFC's without using chlorine atoms. That has been done by reducing the concentration of CFC's partially or totally in the fluids used in the industry. For example, in the refrigeration and air conditioning industry, heat pumps are now filled with the so called environmental friendly refrigerants.

In this work, we will apply the universal equation (HSDS) (Fialho and Nieto de Castro, 1995) to refrigerant mixtures. In order to be able to apply the HSDS model, we use generic mixing and combination rules (Fialho, 1993) for the critical temperature, molar volume, and the relative molecular mass. For the critical pressure the authors use their own rule.

We have applied the HSDS model for mixtures to the binary mixtures of R142b + R22 (Kumagai

\footnotetext{
${ }^{*}$ Corresponding Author.
} 
Table 1

Virial coefficients for the hard-spheres (Ree and Hoover, 1964, Erpenbeck and Wood, 1984)

\begin{tabular}{llll}
\hline$C_{1}$ & 1 & $C_{6}$ & $0.0386 \pm 0.0004$ \\
$C_{2}$ & 1 & $C_{7}$ & $0.0137 \pm 0.006$ \\
$C_{3}$ & $5 / 8$ & $C_{8}$ & 0.00421 \\
$C_{4}$ & 0.28695 & $C_{9}$ & 0.00131 \\
$C_{5}$ & $0.1103 \pm 0.0003$ & $C_{10}$ & 0.00040 \\
\hline
\end{tabular}

et al., 1991, Sousa et al., 1992), R22 + R152a (Maezawa et al., 1991a), R152a + R142b (Maezawa et al., 199lb) and R114 + R22 (Hasegawa et al., 1985, Fukuizumi and Uematsu, 1991). and for the ternary mixture of R22 + R152a + R142b (Maezawa et al., 1991c). The mixtures can act as examples for the test of the model. Except for the binary mixture of R114 + R22 for which we have compressed liquid data, all other mixtures cover only the liquid saturation line. The deviations obtained from these mixtures to the model was better than $1.5 \%$ for $T^{*}=T / T_{\mathrm{c}} \leq 0.9$, which is in good agreement with the values obtained for the pure fluids.

\section{The HSDS reduced model}

As described in a previous paper (Fialho and Nieto de Castro, 1995) the HSDS model (Eq. (1)) has been reduced in order to obtain the universal equation given by expression (2).

$$
\begin{aligned}
& Z=\sum_{i=1}^{10} C_{\mathrm{i}}(b \rho)^{\mathrm{i}-1}-\frac{a \rho}{R T(1+b \rho)} \\
& Z=\sum_{\mathrm{i}=1}^{10} C_{\mathrm{i}}\left(b^{*} \frac{\rho}{\rho_{\mathrm{c}}}\right)^{\mathrm{i}-1}-\frac{a^{*} \frac{p_{\mathrm{c}} \rho}{\rho_{\mathrm{c}}^{2}}}{R T\left(1+b^{*} \frac{\rho}{\rho_{\mathrm{c}}}\right)}
\end{aligned}
$$

where $\rho$ is the molar density, $T$ the temperature in $\mathrm{K}, R$ the gas constant in $\mathrm{J} \mathrm{mol}^{-1} \mathrm{~K}^{-1}, C_{\mathrm{i}}$ are the virial coefficients obtained for the hard sphere model (Table 1), $p_{\mathrm{c}}$ the critical pressure, $\rho_{\mathrm{c}}$ the critical molar density, $a$ and $b$ the attractive and repulsive van der Waals like parameters. Finally $a^{*}$ and $b^{*}$ are the reduced van der Waals like parameters given by Eqs. (3) and (4).

$$
\begin{aligned}
& b^{*}=(2.0335+0.01039 M) \pm 0.0044 \\
& a^{*}=\sum_{i=0}^{2}\left(\sum_{\mathrm{j}=0}^{2} a_{\mathrm{ij}}^{*} T^{* \mathrm{j}}\right) M^{\mathrm{i}}
\end{aligned}
$$

Table 2

Parameters used in Eq. (4)

\begin{tabular}{llll}
\hline$a_{\mathrm{ii}}$ & $\mathrm{i}=0$ & $\mathrm{i}=1$ & $\mathrm{i}=2$ \\
\hline $\mathrm{j}=0$ & $2.069249 \times 10^{5}$ & $-2.332869 \times 10^{6}$ & $9.949903 \times 10^{6}$ \\
$\mathrm{j}=1$ & $-4.245370 \times 10^{5}$ & $5.268112 \times 10^{6}$ & $-2.222515 \times 10^{7}$ \\
$\mathrm{j}=2$ & $2.194730 \times 10^{5}$ & $-2.991949 \times 10^{6}$ & $1.251112 \times 10^{7}$ \\
\hline
\end{tabular}


$M$ is the relative molecular mass of the fluid, $T^{*}$ is the reduced temperature given by $T / T_{\mathrm{c}}$, where $T_{\text {. }}$, is the critical temperature, and $a_{\mathrm{ij}}^{*}$ are given in Table 2.

\section{Mixing and combination rules}

To be able to apply the presented HSDS model to mixtures we have introduced the mixing rules proposed by Reid et al. (1987) for the combination of the properties of the pure components of the mixture in order to obtain its properties. This rule can be expressed to a first approximation by Eq. (5):

$$
Q_{\text {mix }}=\sum_{\mathrm{i}} \sum_{\mathrm{j}} x_{\mathrm{i}} x_{\mathrm{j}} Q_{\mathrm{ij}}
$$

where $Q$ represents the property and $x$ the molar composition of the mixture.

In the present case we have applied this rule to the critical temperature and critical molar volume. For the binary mixture,

$$
\begin{aligned}
& T_{\mathrm{c}_{\text {mix }}}=x_{1}^{2} T_{\mathrm{c}_{1}}+2 x_{1} x_{2} T_{\mathrm{c}_{12}}+x_{2}^{2} T_{\mathrm{c}_{2}} \\
& V_{\mathrm{c}_{\text {mix }}}=x_{1}^{2} V_{\mathrm{c}_{1}}+2 x_{1} x_{2} V_{\mathrm{c}_{12}}+x_{2}^{2} V_{\mathrm{c}_{2}}
\end{aligned}
$$

where the subscripts 1 and 2 stand for components 1 and 2. The subscript 12 represents the cross term. To estimate this term one can use the usual geometric and arithmetic average combination rules (Reid et al., 1987), given by Eqs. (8) and (9).

$$
\begin{aligned}
& T_{\mathrm{c}_{12}}=\sqrt{T_{\mathrm{c}_{1} T_{\mathrm{c}_{2}}}} \\
& V_{\mathrm{c}_{12}}=\left(\frac{V_{\mathrm{c}_{1}}^{1 / 3}+V_{\mathrm{c}_{2}}^{1 / 3}}{2}\right)^{3}
\end{aligned}
$$

To obtain the critical molar density for the mixture one inverts the critical molar volume obtained from Eq. (7). The critical pressure for the mixture is calculated from the critical compressibility factor, obtained from the pure liquid data (Fialho and Nieto de Castro, 1995), and is given by the combination of Eqs. (10) and (11),

$$
\begin{aligned}
& Z_{C}=0.317-\frac{3.41 \times 10^{-3}}{M}-0.138 M \\
& P_{c_{\text {mix }}}=R Z_{\mathrm{c}} \rho_{c_{\text {mix }}} T_{c_{\text {mix }}}
\end{aligned}
$$

where $M$ for the mixtures is given by the linear mixing rule,

$$
M=\sum_{\mathrm{i}} x_{\mathrm{i}} M_{\mathrm{i}}
$$

\section{Discussion}

The reduced state equation of HSDS with the rules presented here has been applied to the binary mixtures of R142b + R22 (Kumagai et al., 1991, Sousa et al., 1992), R22 + R152a (Maezawa et al., 


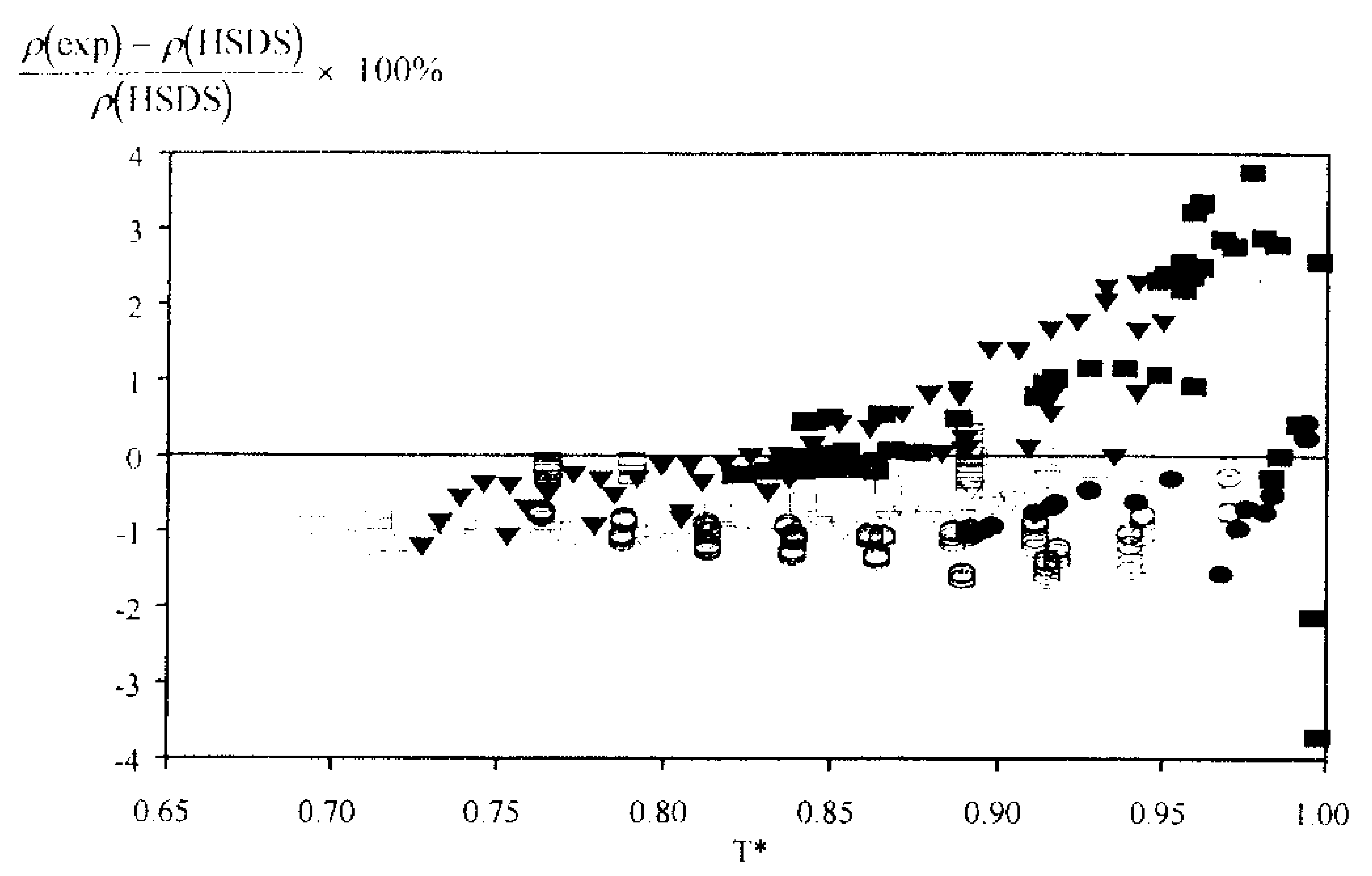

Fig. 1. Deviations between the experimental density, obtained for diferent binary mixtures of refrigerants, and the one predicted by the reduced HSDS model with the combination and mixing rules, Eqs. (5)-(12). $\mathbf{m}, \mathrm{R} 142 \mathrm{~b}+\mathrm{R} 22$ (Kumagai et

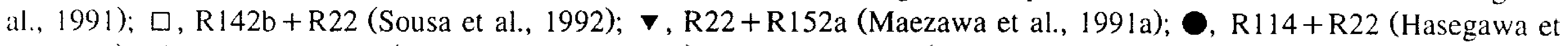
al., 1985); $\nabla, \mathrm{R} 152 \mathrm{a}+\mathrm{R} 142 \mathrm{~b}$ (Maezawa et al., 1991b); $\odot$, R114 + R22 (Fukuizumi and Uematsu, 1991).

1991a), R152a + R142b (Maezawa et al., 1991b) and R114 + R22 (Hasegawa et al., 1985, Fukuizumi and Uematsu, 1991), and for the ternary mixture of R22 + R152a + R142b (Maezawa et al., 1991c). The deviations between the available experimental data and the prediction obtained by the model are presented in Figs. 1 and 2.

We have predicted the density of mixtures for $T^{*} \leq 0.9$ within $1.5 \%$, and better than $2 \%$ for $0.90 \leq T^{*} \leq 0.95$. The calculations covered wide composition ranges, as the data compared for each mixture refers to several compositions. For instance the mixture R152a + R22 involves a composition range from $(10 / 90)$ to $(90 / 10)$. No systematic deviations caused by composition ranges have been detected, although a systematic shift of about $-1 \%$ is encountered for the ternary mixture. This is possibly caused by the need of using more sophisticated mixing or combination rules. However we preferred, for the sake of simplicity, to use a one fluid model for the mixture, predicting its properties from only pure component data and the combination and mixing rules without the use of binary interaction parameters, normally obtained from mixture data.

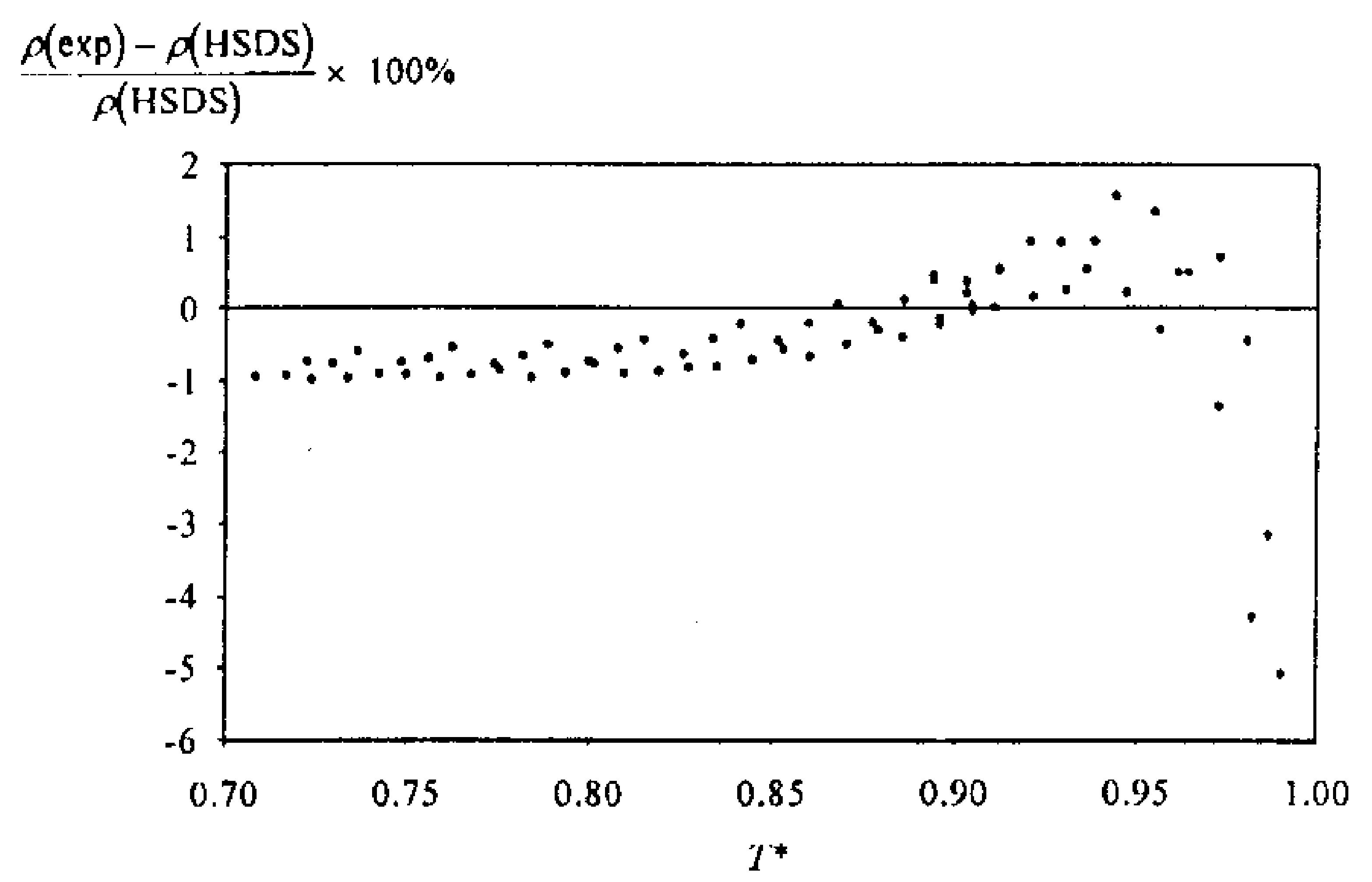

Fig. 2. Deviations between the experimental density of the ternary mixture for R22+R152a+R142b (Maezawa et al., 1991c) and that predicted by the reduced HSDS model with the combination and mixing rules, Eqs. (5)-(12). 
In the companion paper for pure halocarbons (Fialho and Nieto de Castro, 1995) a comparison with the Hankinson-Brobst-Thomson has shown that this equation can produce errors between -4 and $30 \%$. There is no reason to believe that its application to mixtures will give better results. Anther empirical estimation method normally used is the modified Reckett and Hankinson (Reid et al., 1987). However it is only applied to bubble points and uses corrections to mixing rules $\left(k_{\mathrm{ij}}\right)$.

\section{Conclusion}

The present model, together with the mixing rules presented, can be applied to binary and ternary mixtures of refrigerants with an uncertainty of $1.5 \%$ for $T^{*} \leq 0.9$.

As far as the authors can tell, there is no reason one can not apply this model to a mixture of n-components with the same uncertainty. However one should check these assumption carefully, whenever possible.

Since this work was performed, new and accurate dada for the density of more binary and ternary mixtures became available. We hope to report the application of our model to these mixtures in the near future.

\section{References}

Erpenbeck, J.J., Wood, W.W., 1984. Molecular dynamics calculations of the hard sphere equation of state. J. Stat. Phys., 35:
$321-340$.

Fialho, P.S., 1993. Propriedades Termofísicas de Fluidos. Sua Previsão, Estimativa e Correlação. PhD Thesis, University of
Lisbon. Chapter 5.

Fialho, P.S., Nieto de Castro, C.A., 1995. Prediction of halocarbon liquid densities by a modified by Hard Sphere-De Santis equation of state. Fluid Phase Equilibria, in press.

Fukuizumi, H., Uematsu, M., 1991. Thermodynamic properties of R22+R 114 mixtures in the high density region for temperatures from 310 to $370 \mathrm{~K}$. Int. J. Thermophys., 12: 869-876.

Hasegawa, N., Uematsu, M., Watanabe, K., 1985. Measurements of PVTx properties for the R22+R114 system. J. Chem. Eng. Data, 30: 32-36.

Kumagai, K., Yada, N., Sato, H., Watanabe, K., 1991. Measurements of PVTx properties for the binary refrigerant HCFC 142b + HCFC 22 system. J. Chem. Eng. Data, 36: 236-240.

Maezawa, Y., Sato, H., Watanabe, K., 1991a. Saturated liquid densities and bubble-point pressures of the binary HCFC 22 + HFC 152a System. Fluid Phase Equilibria, 61: 263-273.

Maezawa. Y., Widiatmo, J.V., Sato, H., Watanabe, K., 1991b. Saturated liquid densities and bubble-point pressures of the binary HFC 152a + HCFC 142b system. Int. J. Thermophys., 12: 1029-1038.

Maezawa. Y.. Widiatmo, J.V., Sato, H., Watanabe, K., 1991c. Saturated liquid densities and bubble zoint pressures of the ternary HCFC 22/HFC 152a/HCFC 142b system. Fluid Phase Equilibria, 67: 203-212. Ree, F.H., Hoover, W.G., 1964. Fifth and sixth virial coefficients for hard spheres and hard disk.. J. Chem. Phys., 40:
939-950.

Reid, R.C. Prauznitz, J.M., Poling, B.E., 1987. The Properties of Gases \& Liquids, 4th edn., McGraw-Hill. New York, pp. 74-88.

Sousa, A.T., Fialho, P.S., Nieto de Castro, C.A., Tufeu, R., Le Neindre, B., 1992. Density of HCFC $1 \div 2 b$ and of its mixture with HCFC 22. Fluid Phase Equilibria, 80: 213-225. 